\title{
SPACE DEBRIS - THE SHORT TERM ORBITAL \\ EVOLUTION IN THE EARTH GRAVITY FIELD
}

\author{
EDWIN WNUK \\ Astronomical Observatory, A. Mickiewicz University \\ Poznań, Poland
}

\begin{abstract}
Two aspects of the orbital evolution of space debris - the long-term evolution and the short-term one - are of interest for an exploration of the nearEarth space. The paper presents some results concerning the estimation of the accuracy of predicted positions of Earth-orbiting objects for the short-term: a few revolutions or a time-span interval of a few days. Calculations of predicted positions take into account the influence of an arbitrary number of spherical coefficients of the Earth gravity potential. Differences in predicted positions due to differences in the best contemporary geopotential models (JGM-2, JGM-3 and GRIM4-S4) are estimated with the use of an analytical theory of motion and a numerical integration.
\end{abstract}

\section{Introduction}

Intensively explored in the past forty years, the near Earth space is filling up by the constantly increasing number of man-made objects orbiting our planet. These objects can be divided into two categories: satellites which are active or under control, and all other objects - extending in size from dust particles to rocket casings. The second category of objects, called space debris, comprise abandoned satellites, rocket upper stages, objects related to missions (payload, ejected covers, etc.), and objects generated in orbit by fragmentation events. Besides man-made objects orbiting the Earth, a large number of sub-millimetre or millimetre size natural particles - meteoroids - pass through Earth orbital space. The increasing population of objects in the near Earth space present, growing with time, serious hazard for the survival of spacecraft, space stations and astronauts occupying near-Earth orbits. Space debris may significantly disturb any satellite operations or

Celestial Mechanics and Dynamical Astronomy 66: 71-78, 1997.

(C) 1997 Kluwer Academic Publishers. Printed in the Netherlands. 
cause catastrophic damage to a spacecraft. Different techniques, including orbital manoeuvres of a spacecraft, may be applied to protect it from the consequence of collision with a space debris object. In the case of manoeuvres, predicted orbits of the spacecraft and space debris have to be known in advance with sufficient accuracy.

In this paper I discuss the problem of the accuracy of predicted positions of Earth orbiting objects in the case when only an influence of the Earth gravity field on the motion of the objects is taken into account. Among other forces acting on the motion of the Earth orbiting objects, the influence of the non-spherical part of the geopotential is one of the most important and an estimation of uncertainties in predicted orbits of the objects due to uncertainties in modelling of the geopotential is essential.

\section{The Current Population of Space Debris}

The total number of Earth orbiting objects is unknown. Only objects with a diameter larger than about $10 \mathrm{~cm}$ in the low Earth orbits (LEO) and objects larger than $1 \mathrm{~m}$ in the geostationary orbits (GEO) may be permanently tracked by radar or optical facilities and then catalogued. Although the Haystack radar is able to track debris objects as small as $1 \mathrm{~cm}$ at $500 \mathrm{~km}$ altitude and has been used to characterise the orbital debris environment in low Earth orbit (Stansbery et al., 1995), it does not regularly make observations in the frame of the U.S. Space Surveillance Network (SSN). Therefore, objects with a diameter smaller than $10 \mathrm{~cm}$ are not included in the Satellite Catalogue maintained by the U.S. Space Command's Space Surveillance Center. The number of catalogued objects that were in orbit in the middle of 1996 was about 9200 , while the total number of catalogued objects (including decayed objects) was at that time about 25000 and one can observe that these numbers increase constantly with time. Some estimations (e.g. Potter, 1995) show that catalogue population at the lower size $(10 \mathrm{~cm})$ may be incomplete by as much as $50 \%$. Observable objects represent probably only $5-20 \%$ of a population of objects larger than $1 \mathrm{~cm}$, since the number of debris in the $1-10 \mathrm{~cm}$ range is estimated between 35000 and 150000 . The estimated population of particles larger than $1 \mathrm{~mm}$ is more than 1000 times the catalogued population.

\section{Collision Avoidance}

Two Earth orbiting objects can collide with a velocity varying between 0 $\mathrm{km} / \mathrm{s}$ and twice their orbital velocity, approximately $15.4 \mathrm{~km} / \mathrm{s}$ for a low orbit. The average collision velocity, between any spacecraft orbiting in the near-Earth space and debris objects, ranges from about $10 \mathrm{~km} / \mathrm{s}$ for low inclinations of spacecraft orbit, to about $13 \mathrm{~km} / \mathrm{s}$ for near polar orbits. 
Because of high velocity even small particles have sufficient energy to significantly disturb any satellite operations or damage a spacecraft, and debris of the 1-10 cm size range may cause catastrophic damage to a spacecraft. For example, satellite breakup models predict that a $1250 \mathrm{~kg}$ satellite can be catastrophically fragmented by a $1 \mathrm{~kg}$ projectile at $10 \mathrm{~km} / \mathrm{s}$ (Kessler and Loftus, 1995). Applying different shielding techniques, spacecraft may be protected against impacts of space debris with diameters smaller than $1 \mathrm{~cm}$. For larger debris objects, the only effective method to avoid catastrophic consequences of collision between spacecraft and such objects is a manoeuvre that will change the spacecraft orbit. The necessary conditions in this case are the following: 1) the spacecraft must be manoeuvrable, and 2) future positions of the spacecraft and space debris must be known with sufficient accuracy. To be effective, the future positions of debris have to be predicted for a large number of objects (thousands, tens of thousands). For example, in order to protect the International Space Station (orbiting at $450 \mathrm{~km}$ altitude and $28.5 \mathrm{deg}$ inclination) the U.S. Naval Space Command is planning a catalogue of 25000 objects including objects down to $1 \mathrm{~cm}$ in size (Hoots, 1994).

\section{Orbital Evolution of Space Debris}

Two aspects of orbital evolution of the space debris - the long-term evolution and the short-term one - are interesting for an exploration of the near-Earth space. In the case of the long-term evolution (years or tens of years time span) the general characteristics (e.g. total number of objects, spatial distribution and density) of a future space environment are predicted on the basis of the main characteristics of the current population of space debris, and with the use of a relatively simple theory of motion for statistical analysis of future orbits for a large number of objects - a cloud of particles. The long-term evolution of Earth orbiting debris is described in detail by Rossi (1997). In the short-term orbital evolution of space debris objects, considered in this paper, future positions and velocities of individual objects are calculated for a few revolutions or a few days' time span. Osculating elements of orbits, or positions and velocities of a given catalogued object, at the time moment $t$ are predicted with the use of a numerical integration or an analytical theory of motion on the basis of osculating elements at the epoch $t_{0}$, which are derived from tracking data obtained by SSN. Since accuracy requirements concerning predicted orbits are much higher in this case than for the long-term evolution, in calculations one has to take into account different forces acting on the object, besides the central Earth gravity, including the following: the anomalous Earth gravity field, luni-solar attractions, atmospheric drag effects, the so- 
lar radiation pressure, solid Earth and ocean tides and some others effects. Then, predictions for a large number of objects need a great amount of computer time if the numerical integration method is applied. An optimum choice of an analytical theory that saves needed accuracy enables much faster calculations in this case. The accuracy of predicted positions depends mainly on the following: the accuracy of initial conditions (osculating elements), the accuracy of the applied force model, the accuracy of the applied theory (numerical or analytical) and the time interval $\Delta t=t-t_{0}$. Here, I analyse only the influence of uncertainties in the modelling of the Earth gravity on the uncertainties in the object's predicted positions as well as the dependence of the predicted accuracy on the number of geopotential coefficients taken into account in the adopted theory.

\section{The Earth Gravity Field}

Major improvements have been made in the modelling of the Earth gravitational field in the last thirty years. Several geopotential models have been produced on the basis of artificial satellite tracking data and gravimetric measurements. A review of contemporary geopotential models and estimations of their accuracy was presented by Wnuk (1994) and Wnuk and Wilczyńska (1996). The $50 \times 50$ GEM-T3, $70 \times 70$ JGM-2 and JGM$3,70 \times 70$ GRIM4-S4 and $180 \times 180$ WGS-84 models are the ones most frequently used in orbital calculations of artificial Earth satellites. Here, I present (in the next section) results of calculations with the use of the JGM-2 (Nerem et al., 1994), JGM-3 (Tapley et al., 1994) and GRIM4-S4 (Schwinzer et al., 1996) models.

\section{The Accuracy of the Predicted Positions}

Different methods may be used to estimate uncertainties in predicted positions of Earth orbiting objects due to uncertainties in the modelling of disturbing forces, particularly in modelling of the geopotential. The method used here is based on the comparison of the predicted positions of the given object calculated with the use of the same initial conditions, the same theory of motion and different geopotential models. Differences in the predicted positions, following the differences in the models of the Earth's gravity are an estimation of uncertainties in the determination of future positions of a spacecraft and space debris, and thus also an estimation of the prediction accuracy of a collision between the spacecraft and space debris.

The predicted orbits were calculated by the system of computer programs prepared on the basis of theories of an artificial satellite motion developed in the Astronomical Observatory of the A. Mickiewicz University, Poznań, Poland (Wnuk, 1988; Wnuk, 1995; Wnuk and Breiter, 1990). 


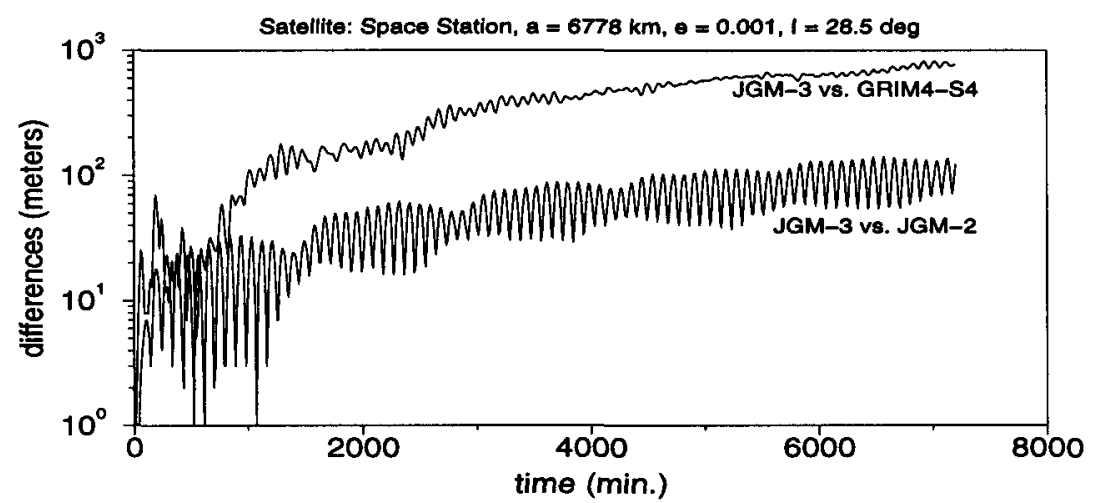

Figure 1. Differences in predicted positions for the Space Station orbit calculated with the use of different Earth's gravity models.

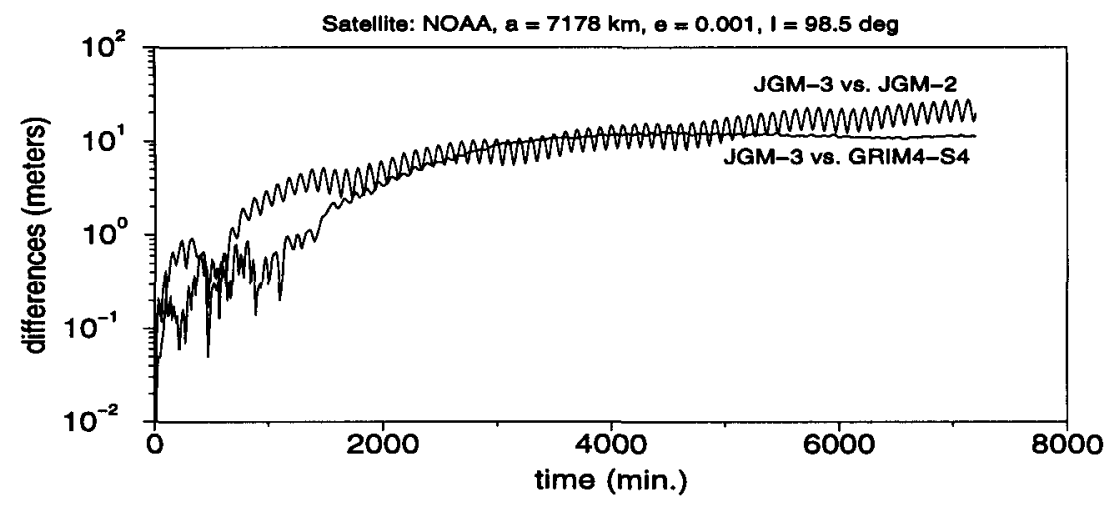

Figure 2. The same as on the Figure 1 for the NOAA orbit.

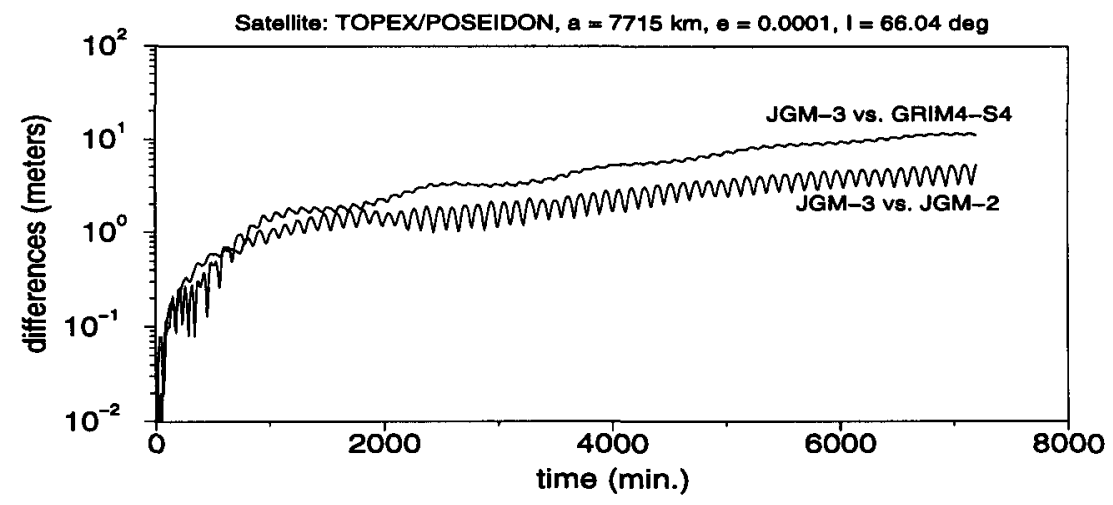

Figure 3. The same as on the Figure 1 for the TOPEX/POSEIDON orbit. 


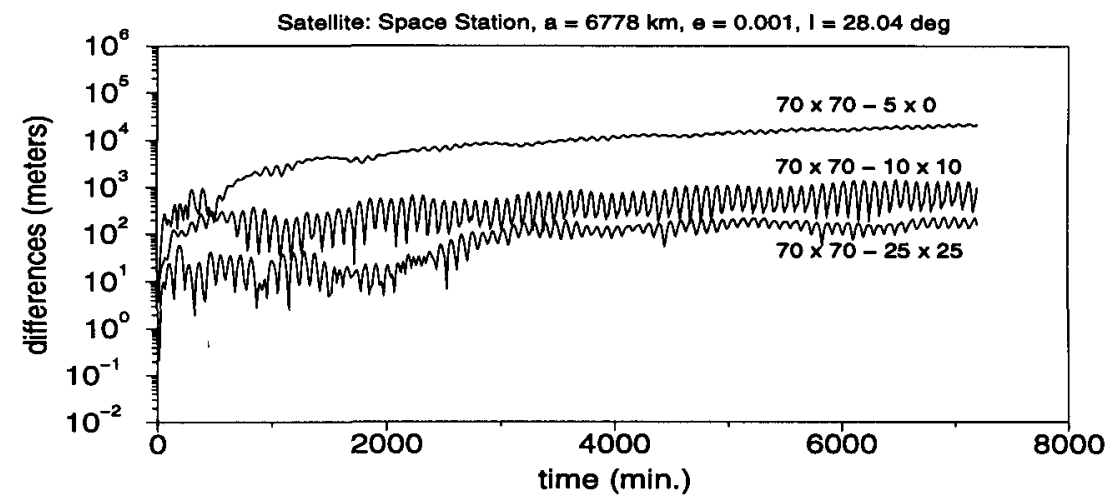

Figure 4. Differences in predicted positions for the Space Station orbit calculated with the use of different number of geopotential coefficients.

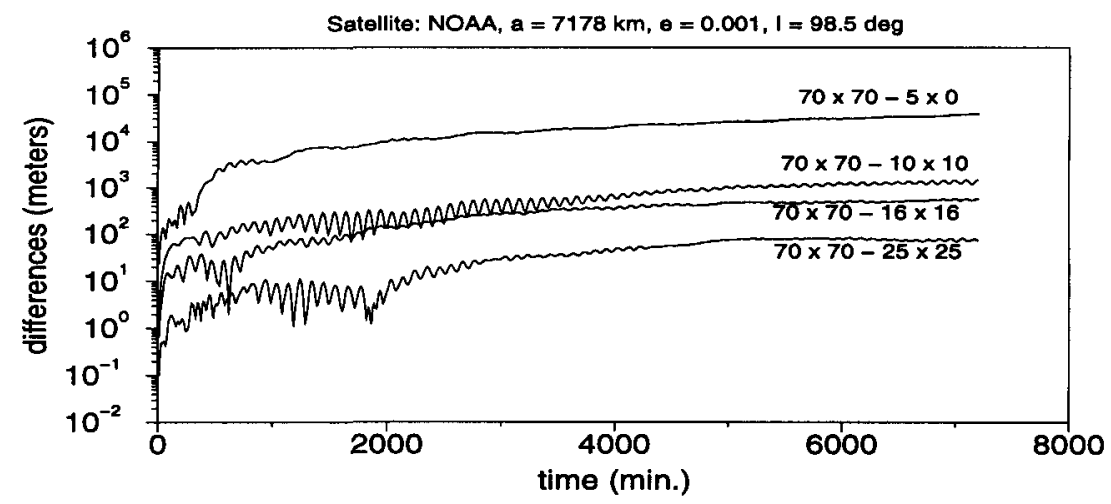

Figure 5. The same as on the Figure 4 for the NOAA orbit.

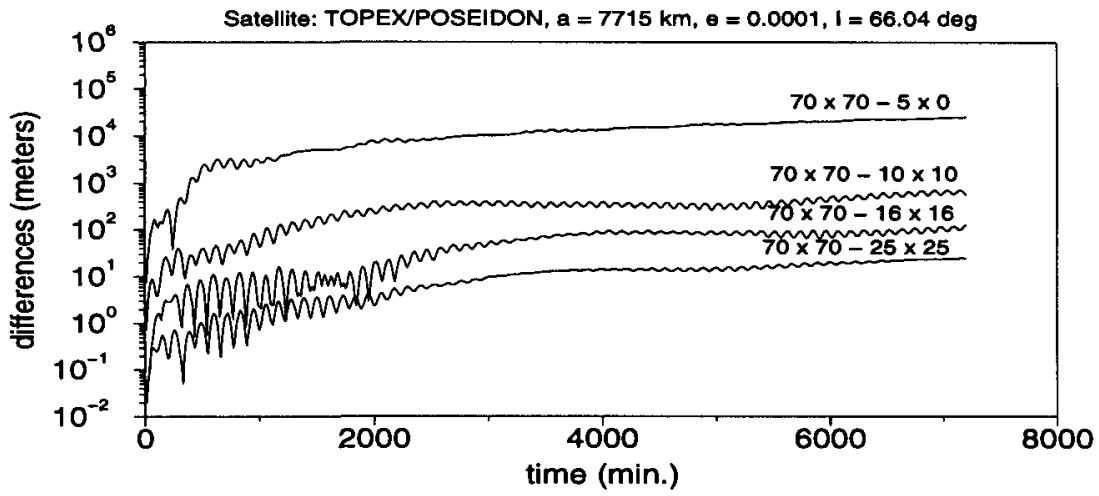

Figure 6. The same as on the Figure 4 for the TOPEX/POSEIDON orbit. 
This system enables the precise calculation of orbits, perturbations in components of the radius vector, and predicted positions of the Earth-orbiting objects with the application of an arbitrary degree and order of geopotential spherical coefficients. The results obtained with the use of the analytical theory of motion were comparable with the results obtained from numerical integration of equations of motion on a level of the accuracy of the analytical theory. Differences in predicted positions for the Space Station orbit calculated with the use of all spherical harmonic coefficients of the JGM-3, JGM-2 and GRIM4-S4 geopotential models for a 5-day time interval are plotted in Figure 1. Similar differences for the NOAA satellites orbits and the TOPEX/POSEIDON orbit are plotted in Figures 2 and 3, respectively. One can see that the differences in the best contemporary Earth gravity models produce differences in the predicted position of orbiting objects on the level of $100-400$ meters for very low orbits and on the level of $10-$ 20 meters for the TOPEX/POSEIDON orbit. After a 1 day time interval, which is needed for preparing the Space Station manoeuvre, the differences are on the level of about 100 meters. These differences essentially depend not only on the orbit's altitude but also on the inclination (Wnuk and Wilczyńska, 1996). Uncertainties in predicted positions are much higher for orbits with a low inclination.

Another question concerning the accuracy of predictions is: how many spherical coefficients one has to include in the calculations to save a given level of accuracy. Precise orbit calculations must take into account all coefficients of the applied geopotential model (e.g. more than $\mathbf{5 0 0}$ for the JGM-3). Since algorithms are complex and time consuming in this case, in practice algorithms include only certain of the coefficients, all others being omitted. For example, the SPG4 algorithm used by the U.S. Space Command includes only J2 - J4. Figures $4-6$ present differences between predicted satellite positions calculated with the use of all coefficients of the JGM-3 model up to $70 \times 70$ and positions calculated with the use of only certain coefficients. We see that to save 100 meter accuracy one has to include coefficients up to $25^{\text {th }}$ degree and order for the Space Station orbit and coefficients up to $16^{\text {th }}$ degree and order for the NOAA and the TOPEX/POSEIDON orbits.

\section{Conclusions}

Space debris can seriously restrict space activities and even an access to space. One of the ways to avoid the consequences of collisions between an operating spacecraft and space debris objects is a manoeuvre changing the spacecraft orbit. The necessary condition in this case is the knowledge of predicted positions of the spacecraft and the debris. The accuracy requi- 
rement of predictions depends on the spacecraft and its manoeuvrability. Predictions must be sufficiently accurate to avoid false alarms and to provide high confidence of predicted collisions. The predicted accuracy is limited by an observation (tracking) accuracy, restricted accuracy of the force model and limitation in the adopted theories. In the case of a large number of objects the time of computation is critical and the algorithms for the predictions should be optimised by introducing new analytical theories and an application of parallel processing.

Acknowledgements. This paper has been supported by the Polish Committee for Scientific Research under the grant No 2 PO3C.006.08.

\section{References}

Hoots, F.R.: 1994, "The need for new algorithms", Proceedings of the Artificial Satellite Theory Workshop (P.K. Seidelmann, B. Kaufman, eds), U.S. Naval Observatory, Washington.

Kessler, D.J. and Loftus, J.P.: 1995, "Orbital debris as an energy management problem", Adv. Space Res. 16, (11)139-(11)144.

Nerem, R.S., Lerch, F.J., Marschall, J.A., Pavlis, E.C., Putney, B.H., Tapley, B.D., Eanes, R.J., Ries, J.C., Schutz, B.E., Shum, C.K., Watkins, M.M., Chan, J.C., Klosko, J.M., Luthcke, S.B., Patel, G.B., Pavlis, N.K., Williamson, R.G., Rapp, R.H., Biancale, R., and Nouel, F.: 1994, "Gravity model development for TOPEX/POSEIDON: Joint gravity models 1 and 2", J. Geophys. Res. 99 (C12), 24421-24448.

Potter, A.E.: 1995, "Ground-based optical observations of orbital debris: A reviev", Adv. Space Res. 16, (11)35-(11)45.

Rossi, A.: 1997, "Long term evolution of Earth orbiting debris", this volume.

Schwinzer, P., Reigber, Ch., Bode, A., Kang, Z., Zhu, S.Y., Massmann, F.-H., Raimondo, J.C., Biancale, R., Balmino, G., Lemoine, J.M., Moynot, B., Marty, J.C., Barlier, F., and Boudon, Y.: 1996, "Long-wavelength global gravity field models: GRIM4-S4, GRIM4-C4", J. Geod., in print.

Stansbery, E.G., Kessler, D.J., Tracy, T.E., Matney, M.J., and Stanley, J.F.: 1995, "Characterization of the orbital debris environment from Haystack radar measurements", Adv. Space Res. 16, (11)5-(11)16.

Tapley, B.D.,Watkins, M.M., Ries, J.C., Davis, G.W., Eanes, R.J., Poole, S.R., Rim, H.J., Schutz, B.E., Shum, C.K., Nerem, R.S., Lerch, F.J., Pavlis, E.C., Klosko, S.M., Pavlis, N.K., and Williamson, R.C., 1994, "The JGM-3 gravity model", Ann. Geophys. 12, Suppl. 1, C192.

Wnuk, E.: 1988, "Tesseral harmonic perturbations for high order and degree harmonics", Celest. Mech. 44, 179-191.

Wnuk, E.: 1994, "Earth gravity field and geopotential perturbations of the Earth's artificial satellites", Proceedings of the Artificial Satellite Theory Workshop (P.K. Sejdelmann, B. Kaufman, eds), U.S. Naval Observatory, Washington.

Wnuk, E.: 1995, "Second order perturbations due to the gravity potential of a planet", in: From Newton to Chaos (A.E. Roy, B.A. Steves, eds), Plenum Press, New York.

Wnuk, E. and Breiter, S.: 1990, "Tesseral harmonic perturbations in radial, transverse and binormal components", Celest. Mech. 48, 375-385.

Wnuk, E. and Wilczyńska, E.: 1996, "The accuracy of contemporary geopotential models and the accuracy of predicted positions of artificial Earth satellites", in: Global Gravity Field and its Temporal Variations, IAG Symposium 116 (R.H. Rapp, A.A. Cazenave, R.S. Nerem, eds), Springer. 